\title{
Varieties of Parametric Classes of Exact Solutions in General Relativity Representing Static Fluid Balls
}

\author{
Neeraj Pant \\ Department of Mathematics, National Defence Academy, Khadakwasla, Pune 411023, India \\ Correspondence should be addressed to Neeraj Pant, neeraj.pant@yahoo.com \\ Received 4 November 2011; Accepted 18 December 2011 \\ Academic Editor: H. Zhao
}

Copyright () 2011 Neeraj Pant. This is an open access article distributed under the Creative Commons Attribution License, which permits unrestricted use, distribution, and reproduction in any medium, provided the original work is properly cited.

We have presented a method of obtaining parametric classes of spherically symmetric analytic solutions of the general relativistic field equations in canonical coordinates. A number of previously known classes of solutions have been rediscovered which describe perfect fluid balls with infinite central pressure and infinite central density though their ratio is positively finite and less than one. From the solution of one of the newly discovered classes, we have constructed a causal model in which outmarch of pressure and density is positive and monotonically decreasing, and pressure-density ratio is positive and less than one throughout within the balls. Corresponding to this model, we have maximized the Neutron star mass $2.40 M_{\Theta}$ with the linear dimensions of $28.43 \mathrm{kms}$ and surface red shift of 0.4142 .

\section{Introduction}

Due to nonlinearity of the Einstein's field equations, many attempts have been reported to obtain parametric classes of exact solutions of representing perfect fluid ball in equilibrium such as Tolman [1], Wyman [2], Kuchowich [3], Pant and Sah [4], D.N. Pant and N. Pant [5], Pant [6, 7], Pant [8], Delgaty, Lake [9], Lake [10], Tewari and Pant [11], and Maurya, Gupta [12]. These solutions have four arbitrary constants. The usual boundary conditions determine three arbitrary constants leaving one undetermined; such a solution represents a class of solutions, with undetermined constant being a parameter. The edge of a parametric class of solutions over an ordinary solution lies in the choice of associated parameter which provides us various models of relativistic stars with realistic equation of state. Moreover, by imposing realistic conditions on parameter, one may get physical and significant solutions. Thus in the light of importance of parametric class, in this paper, we present a variety of parametric classes of solutions.

\section{Field Equations and Method of Obtaining Parametric Classes of Solutions}

We consider the static and spherically symmetric metric in canonical coordinates

$$
d s^{2}=-e^{\lambda} d r^{2}-r^{2}\left(d \theta^{2}+\sin ^{2} \theta d \phi^{2}\right)+e^{v} d t^{2},
$$

where $\lambda$ and $v$ are field variables and functions of $r$ only. The field equations of gravitation for a nonempty space-time are

$$
R_{i j}-\frac{1}{2} R g_{i j}=-\frac{8 \pi G}{c^{4}} T_{i j},
$$

where $R_{i j}$ is a Ricci tensor, $T_{i j}$ is energy-momentum tensor, and $R$ the scalar curvature. The energy-momentum tensor $T_{i j}$ is defined as

$$
T_{i j}=\left(p+\rho c^{2}\right) v_{i} v_{j}-p g_{i j},
$$

where $p$ denotes the pressure distribution, $\rho$ the density distribution, and $v_{i}$ the velocity vector, satisfying the relation

$$
g_{i j} v^{i} v^{j}=1 \text {. }
$$


Since the field is static, therefore, only a nonzero component of velocity is $v^{4}$

$$
v^{4}=\frac{1}{\sqrt{g_{44}}} .
$$

Thus, under these conditions, the field equations of general relativity for a perfect fluid ball the physical variables $p(r)$ and $\rho(r)$ are (Tolman[1])

$$
\begin{gathered}
\frac{8 \pi G}{c^{4}} p=e^{-\lambda}\left(\frac{v^{\prime}}{r}+\frac{1}{r^{2}}\right)-\frac{1}{r^{2}}, \\
\frac{8 \pi G}{c^{2}} \rho=e^{-\lambda}\left(\frac{\lambda^{\prime}}{r}-\frac{1}{r^{2}}\right)+\frac{1}{r^{2}}, \\
\frac{d}{d r}\left(\frac{e^{-\lambda}-1}{r^{2}}\right)+\frac{d}{d r}\left(\frac{e^{-\lambda} v^{\prime}}{2 r}\right)+e^{-\lambda-v} \frac{d}{d r}\left(\frac{e^{v} v^{\prime}}{2 r}\right)=0,
\end{gathered}
$$

where prime (/) denotes differentiation with respect to $r$. The problem consists of solving (8) for $\lambda$ and $v$ and obtaining $p(r)$ and $\rho(r)$ from (6) and (7).

By using the transformation

$$
U=r^{m-1} e^{m v / 2}, \quad V=e^{-\lambda} .
$$

Equation (8) reduces to the following linear differential equation in $V$ :

$$
\begin{aligned}
\frac{d V}{d r} & -2\left\{\frac{d}{d r}\left\{\log \left(\frac{r^{4-1 / m} U^{1-1 / m}}{r U^{\prime}+U}\right)\right\}-\frac{2 m U}{r\left(r U^{\prime}+U\right)}\right\} V \\
& =\frac{-2 m U}{r\left(r U^{\prime}+U\right)} .
\end{aligned}
$$

On solving (10), we get

$$
V=e^{-\lambda}=\frac{r^{8-2 / m} U^{2(1-1 / m)}}{\left(r U^{\prime}+U\right)^{2}}\left[A-2 m \int \frac{\left(r U^{\prime}+U\right) U^{-1+2 / m} e^{\int\left(4 m U d r / r\left(r U^{\prime}+U\right)\right)}}{r^{9-2 / m}} d r\right] e^{-\int\left(4 m U d r / r\left(r U^{\prime}+U\right)\right)},
$$

where " $A$ " is an arbitrary constant. Our aim is to explore the possibilities of choosing $U$ such that the right-hand side of (11) becomes integrable. In this paper, we assume

$$
e^{\int\left(4 m U d r / r\left(r U^{\prime}+U\right)\right)}=r^{l}\left(r U^{\prime}+U\right)^{n}
$$

$l, m$, and $n$ are arbitrary constants. Equation (12) results into a second degree homogenous differential equation in $\mathrm{U}$

$$
n r^{2} U^{/ /}+(l+2 n) r U^{\prime}+(l-4 m) U=0 .
$$

The solution is

$$
U=C_{1} r^{a+b-1}+C_{2} r^{a-b-1},
$$

where

$$
a=\frac{n-l}{2 n}, \quad b=\frac{1}{2 n} \sqrt{(n-l)^{2}+16 m n,}
$$

provided, $n \neq 0$.

$C_{1}$ and $C_{2}$ are arbitrary constants. Also (11) is simplified into

$$
\begin{aligned}
V & =e^{-\lambda} \\
& =\frac{r^{8+n-l-(a-b)(n+2 / m)}[A-2 I]}{\left[(a+b) C_{1} r^{2 b}+(a-b) C_{2}\right]^{n+2}\left(C_{1} r^{2 b}+C_{2}\right)^{2(-1+1 / m)}},
\end{aligned}
$$

where

$$
\begin{aligned}
I \equiv m \int r^{l-n-9+(a-b)(n+2 / m)}\left[(a+b) C_{1} r^{2 b}+(a-b) C_{2}\right]^{n+1} \\
\times\left[C_{1} r^{2 b}+C_{2}\right]^{-1+2 / m} d r .
\end{aligned}
$$

The solution is complete if (17) is integrated. In the foregoing sections, we shall discuss the method of solving (17), which will result into the variety of classes of solutions.

\section{Varieties of Classes of Solutions}

To explore the integrability of (17), we substitute

$$
\begin{gathered}
r^{2 b}=y, \\
l-n-9+(a-b)\left(n+\frac{2}{m}\right)=2 b \alpha-1 .
\end{gathered}
$$

So that (17) transforms into

$$
\begin{aligned}
I \equiv \frac{m}{2 b} \int & y^{\alpha-1}\left[(a+b) C_{1} y+(a-b) C_{2}\right]^{n+1} \\
& \times\left[C_{1} y+C_{2}\right]^{-1+2 / m} d y .
\end{aligned}
$$

It is easy to see that right-hand side of (20) can be integrated by parts with the restriction on the exponents of integrand to be nonnegative integral values. Thus we arrive at varieties of classes of solutions.

3.1. Class I (new class of solutions). We assume $n+1=0$ and $-1+2 / m=0$. In view of (18) and (20), the resulting class of solutions is

$$
\begin{gathered}
e^{v}=\left(C_{1} r^{a+b-2}+C_{2} r^{a-b-2}\right), \\
e^{-\lambda}=\frac{\left[A r^{7-l}-4 /(l-7)\right]\left[C_{1} r^{2 b}+C_{2}\right]}{\left[(a+b) C_{1} r^{2 b}+(a-b) C_{2}\right]},
\end{gathered}
$$


where

$$
a=\frac{1+l}{2}, \quad b=\frac{\sqrt{(1+l)^{2}-32}}{-2} .
$$

We observe that $\left(e^{v}\right)_{r=0}$ becomes singular for all values except $\sqrt{32}-1 \leq l \leq 5$. It may be pointed out here that for $l=5$, we rediscover Tolman's IV solution which is the only member of the class giving rise to finite central pressure and central density.

\section{Properties of the New Class of Solutions}

In view of (??), we obtain from (6) and (7), the pressure and density distribution, respectively,

$$
\begin{gathered}
\frac{8 \pi G}{c^{4}} p=\frac{1}{r^{2}}\left\{\frac{\left[A r^{7-l}-4 / l-7\right]\left[(a+b-1) C_{1} r^{2 b}+(a-b-1) C_{2}\right]}{\left[(a+b) C_{1} r^{2 b}+(a-b) C_{2}\right]}-1\right\}, \\
\frac{8 \pi G}{c^{2}} \rho=\frac{1}{r^{2}}\left[\frac{\left[\left(A r^{7-l}-4 / l-7\right) 4 b^{2} C_{1} C_{2} r^{2 b}+\left((l-8) A r^{7-l}+4 / l-7\right) \times\left(C_{1} r^{2 b}+C_{2}\right)\left((a+b) C_{1} r^{2 b}+(a-b) C_{2}\right)\right]}{\left[(a+b) C_{1} r^{2 b}+(a-b) C_{2}\right]^{2}}+1\right] .
\end{gathered}
$$

The central values of pressure and density are infinite; however, the limiting value of their ratio is finite and equal to the limiting value of $d p / d \rho$ :

$$
\begin{aligned}
\left(\frac{p}{\rho c^{2}}\right)_{r \rightarrow 0} & =\frac{1}{c^{2}}\left(\frac{d p}{d \rho}\right)_{r \rightarrow 0} \\
& =-\frac{[4(a+b-1)+(l-7)(a+b)]}{[4+(l-7)(a+b)]} .
\end{aligned}
$$

The causality condition at the centre is valid for all those values of $l$ satisfying the inequality $\sqrt{32}-1 \leq l \leq 5$.

In addition to the parameter $l$, the solutions (??) contain three arbitrary constants $C_{1}, C_{2}$, and $A$. These are to be determined by matching the solutions (??) with Schwarzschild exterior solution for a ball of mass $M$ and linear dimension $2 r_{b}$ :

$$
\begin{gathered}
p\left(r_{b}\right)=0, \\
e^{-\lambda\left(r_{b}\right)}=1-2 u, \\
e^{v\left(r_{b}\right)}=1-2 u,
\end{gathered}
$$

where

$$
u=\frac{G M}{c^{2} r_{b}}
$$

Consequently,

$$
\begin{gathered}
C_{1}=-\frac{[(a-b-1)(1-2 u)-1]}{2 b r_{b}^{a+b-2}}, \\
C_{2}=\frac{[(a+b-1)(1-2 u)-1]}{2 b r_{b}^{a-b-2}}, \\
A=r_{b}^{l-7}\left[\frac{[1-2 u]\left[(a+b) C_{1} r_{b}^{2 b}+(a-b) C_{2}\right]}{\left[C_{1} r_{b}^{2 b}+C_{2}\right]}+\frac{4}{l-7}\right] .
\end{gathered}
$$

For $e^{v}$ to be definitely positive in the region $0 \leq r \leq r_{b}$, we must have $C_{1}, C_{2} \geq 0$. Thus in view of (27) and (28), we have

$$
\frac{a+b-2}{a+b-1} \leq 2 u \leq \frac{a-b-2}{a-b-1} .
$$

\section{Particular Member of Class I for $l=4.75$}

In this section, we shall present a detail study of the particular solution corresponding to $l=4.75$.

The solution is

$$
\begin{gathered}
e^{v}=\left(C_{1} r^{0.35962}+C_{2} r^{1.39}\right), \\
e^{-\lambda}=\frac{\left[A r^{2.25}+1.78\right]\left[C_{1}+C_{2} r^{1.03}\right]}{\left[2.359 C_{1}+3.39 C_{2} r^{1.03}\right]}, \\
\frac{8 \pi G}{c^{4}} p=\frac{1}{r^{2}}\left[\frac{\left[A r^{2.25}+1.78\right]\left[1.359 C_{1}+2.39 C_{2} r^{1.03}\right]}{\left[2.359 C_{1}+3.39 C_{2} r^{1.03}\right]}-1\right], \\
\frac{8 \pi G}{c^{2}} \rho=\frac{1}{r^{2}}\left[\frac{\left[\left(A r^{2.25}+1.78\right) 1.06 C_{1} C_{2} r^{1.03}+\left(-3.25 A r^{2.25}-1.78\right) \times\left(C_{1}+C_{2} r^{1.03}\right)\left(2.359 C_{1}+3.39 C_{2} r^{1.03}\right)\right]}{\left[2.359 C_{1}+3.39 C_{2} r^{1.03}\right]^{2}}+1\right] .
\end{gathered}
$$


TABLE 1: The march of pressure, density, pressure-density ratio, and square of adiabatic sound speed within the ball corresponding to $l=4.75$ with $u=0.25$.

\begin{tabular}{lcccc}
\hline$r / r_{b}$ & $\left(8 \pi G / c^{4}\right) p r_{b}^{2}$ & $\left(8 \pi G / c^{2}\right) \rho r_{b}^{2}$ & $p / \rho c^{2}$ & $\left(1 / c^{2}\right)(d p / d \rho)$ \\
\hline 0 & $\infty$ & $\infty$ & 0.09879 & 0.09879 \\
0.1 & 6.58927 & 32.3632 & 0.20360 & 0.18684 \\
0.2 & 2.29996 & 9.40575 & 0.24452 & 0.23125 \\
0.3 & 1.18659 & 4.63777 & 0.25585 & 0.26815 \\
0.4 & 0.70315 & 2.84393 & 0.24724 & 0.29885 \\
0.5 & 0.44151 & 1.97301 & 0.22377 & 0.32653 \\
0.6 & 0.28097 & 1.48486 & 0.18922 & 0.36966 \\
0.7 & 0.17397 & 1.18389 & 0.14694 & 0.39023 \\
0.8 & 0.09830 & 0.98631 & 0.09966 & 0.42714 \\
0.9 & 0.04233 & 0.84997 & 0.049801 & 0.46666 \\
1.0 & 0 & 0.75238 & 0 & 0.45254 \\
\hline
\end{tabular}

In view of $(30) e^{v}$ to be definitely positive in the region $0 \leq$ $r \leq r_{b}$, then

$$
0.13225 \leq u \leq 0.29079 .
$$

Corresponding to $u=0.25$ and in view of (27), (28), and (29), the constants are

$$
\begin{gathered}
C_{1} r_{b}^{0.35962}=0.1891, \quad C_{2} r_{b}^{1.39}=0.3106, \\
A r_{b}^{2.25}=-0.2808 .
\end{gathered}
$$

In Table 1 the march of pressure, density, pressuredensity ratio, and square of adiabatic sound speed $d p / d \rho$ is given for $u=0.25$. We observe that pressure and density decrease monotonically with the increase of radial coordinate, pressure-density ratio, and square of adiabatic sound speed, which is positive and less than 1 throughout within the ball.

We now present here a model of Neutron star based on the particular solution discussed above. The Neutron star is supposed to have a surface density: $\rho_{b}=2 \times 10^{14} \mathrm{gm}-\mathrm{cm}^{-3}$ suggested by Brecher and Caporaso [13]. The resulting causal model has the mass $M=2.40 M_{\Theta}$ and the linear dimension $2 r_{b} \approx 28.43 \mathrm{~km}$. The surface red shift $Z_{b}=\left[(1-2 u)^{-0.5}-\right.$ $1] \approx 0.4142$.

\section{Some More Parametric Classes of Solutions}

By assigning the different nonnegative integral values to the exponents of (20), we obtain the following different parametric classes of solutions.

6.1. Class II. If we assume $\alpha-1=0$ and $-1+2 / m=0$. In view of (17), (18), and (20), the resulting class of solutions is (see Neeraj Pant [6])

$$
e^{v}=\left(C_{1} r^{a+b-2}+C_{2} r^{a-b-2}\right),
$$

$$
\begin{aligned}
e^{-\lambda}= & {\left[\frac{A r^{-2 b}}{\left[(a+b) C_{1} r^{2 b}+(a-b) C_{2}\right]^{n+2}}\right.} \\
& \left.-\frac{r^{-2 b}}{2 b(n+2)(a+b) C_{1}}\right]\left[C_{1} r^{2 b}+C_{2}\right] .
\end{aligned}
$$

6.2. Class III. If we assume $\alpha-1=0$ and $n+1=0$. In view of (17), (18), and (20), the resulting class of solutions is (see Pant $[8])$

$$
\begin{aligned}
e^{v} & =\left(C_{1} r^{a+b-m}+C_{2} r^{a-b-m}\right)^{2 / m}, \\
e^{-\lambda} & =\left[\frac{A r^{-2 b}-m^{2} r^{-2 b} / 2 b C_{1}\left(C_{1} r^{2 b}+C_{2}\right)^{2 / m}}{\left[(a+b) C_{1} r^{2 b}+(a-b) C_{2}\right]\left(C_{1} r^{2 b}+C_{2}\right)^{2(-1+1 / m)}}\right] .
\end{aligned}
$$

6.3. Class $I V$. If we assume $n+1=0$ and $-1+2 / m=1$. In view of (17), (18), and (20), the resulting class of solutions is (see Pant $[8]$ )

$$
\begin{aligned}
e^{v} & =\left(C_{1} r^{a+b-1}+C_{2} r^{a-b-1}\right)^{2}, \\
e^{-\lambda} & =\frac{r^{-2 b \alpha}\left[A-r^{2 b \alpha}\left\{\alpha C_{1} r^{2 b}+(\alpha+1) C_{2}\right\} / b \alpha(\alpha+1)\right]}{\left[(a+b) C_{1} r^{2 b}+(a-b) C_{2}\right]},
\end{aligned}
$$

where

$$
\alpha=\frac{l-7+a-b}{2 b} .
$$


6.4. Class $V$. If we assume $\alpha-1=1$ and $-1+2 / m=0$. In view of (17), (18), and (20), the resulting class of solutions is (New Class of Solutions)

$$
\begin{aligned}
e^{v} & =\left(C_{1} r^{a+b-2}+C_{2} r^{a-b-2}\right), \\
e^{-\lambda} & =\frac{A r^{-4 b}-4 B r^{-4 b} \mathfrak{A} \mathfrak{B}}{\left[(a+b) C_{1} r^{2 b}+(a-b) C_{2}\right]^{n+2}\left(C_{1} r^{2 b}+C_{2}\right)^{-1}},
\end{aligned}
$$

where $\mathfrak{A}$ denotes $\left[(a+b) C_{1} r^{2 b}+(a-b) C_{2}\right]^{n+2}$ and $\mathfrak{B}$ denotes $\left[(a+b)(n+2) C_{1} r^{2 b}-(a-b) C_{2}\right]$,

$$
B=\frac{1}{b(a+b)^{2}(n+2)(n+3) C_{1}{ }^{2}} \text {. }
$$

6.5. Class VI. If we assume $\alpha-1=1$ and $n+1=0$. In view of (17), (18), and (20), the resulting class of solutions is (New Class of Solutions)

$$
\begin{aligned}
e^{v} & =\left(C_{1} r^{a+b-m}+C_{2} r^{a-b-m}\right)^{2 / m}, \\
e^{-\lambda} & =\left[\frac{\mathfrak{D}\left(2 C_{1} r^{2 b}-m C_{2}\right)}{\left[(a+b) C_{1} r^{2 b}+(a-b) C_{2}\right]\left(C_{1} r^{2 b}+C_{2}\right)^{2(-1+1 / m)}}\right],
\end{aligned}
$$

where $\mathfrak{D}$ denotes $A r^{-4 b}-2 m^{2} r^{-4 b} / 4 b C_{1}{ }^{2}\left(C_{1} r^{2 b}+C_{2}\right)^{2 / m}$.

6.6. Class VII. If we assume $\alpha-1=0$ and $-1+2 / m=1$. In view of (17), (18), and (20), the resulting class of solutions is (see Tewari and Pant [11])

$$
\begin{aligned}
e^{v} & =\left(C_{1} r^{a+b-1}+C_{2} r^{a-b-1}\right)^{2}, \\
e^{-\lambda} & =\frac{A r^{-2 b}}{\left[(a+b) C_{1} r^{2 b}+(a-b) C_{2}\right]^{n+2}}-B\left(C_{1}^{\prime}+C_{2}^{\prime} r^{-2 b}\right) .
\end{aligned}
$$

\section{Conclusion}

By assigning different nonnegative integral values to the any two pairs of exponents of integrand of (17), that is, $\alpha-1, n+1,-1+2 / m$, we arrive at different parametric classes of solutions. All these classes have of the form $e^{v}=\left(C_{1} r^{\beta}+C_{2} r^{\beta}\right)^{\gamma}$. For meaningful solutions $\gamma, \beta$ to be nonnegative, thus restriction on parameter ranges.

\section{Acknowledgments}

The author expresses his gratitude to Professer O. P. Shukla, Principal NDA, for his encouragement. He is also grateful to the anonymous referees for pointing out the errors in the original paper and making constructive and relevant suggestions.

\section{References}

[1] R. C. Tolman, "Static solutions of einstein's field equations for spheres of fluid," Physical Review, vol. 55, no. 4, pp. 364-373, 1939.

[2] M. Wyman, "Radially symmetric distributions of matter," Physical Review, vol. 75, no. 12, pp. 1930-1936, 1949.

[3] B. Kuchowicz, "General relativistic fluid spheres. I. New solutions for spherically symmetric matter distributions," Acta Physica Polonica, vol. 33, pp. 541-563, 1968.

[4] D. N. Pant and A. Sah, "Class of solutions of Einstein's field equations for static fluid spheres," Physical Review D, vol. 26, no. 6, pp. 1254-1261, 1982.

[5] D. N. Pant and N. Pant, "A new class of exact solutions in general relativity representing perfect fluid balls," Journal of Mathematical Physics, vol. 34, no. 6, pp. 2440-2447, 1993.

[6] N. Pant, "Uniform radial motion of sound in a relativistic fluid ball," Astrophysics and Space Science, vol. 240, no. 2, pp. 187193, 1996.

[7] N. Pant, "Some new exact solutions with finite central parameters and uniform radial motion of sound," Astrophysics and Space Science, vol. 331, no. 2, pp. 633-644, 2011.

[8] D. N. Pant, "Varieties of new classes of interior solutions in general relativity," Astrophysics and Space Science, vol. 215, no. 1, pp. 97-109, 1994.

[9] M. S.R. Delgaty and K. Lake, "Physical acceptability of isolated, static, spherically symmetric, perfect fluid solutions of Einstein's equations," Computer Physics Communications, vol. 115, no. 2-3, pp. 395-415, 1998.

[10] K. Lake, "All static spherically symmetric perfect-fluid solutions of Einstein's equations," Physical Review D, vol. 68, no. $10,2003$.

[11] B. C. Tewari and M. Pant, "New parametric classes of exact solutions in general relativity and polytropic nature fluid ball with constant sound speed," Astrophysics and Space Science, vol. 332, no. 2, pp. 409-414, 2011.

[12] S. K. Maurya and Y. K. Gupta, "A family of physically realizable perfect fluid spheres representing a quark-stars in general relativity," Astrophysics and Space Science. In press.

[13] K. Brecher and G. Caporaso, "Obese neutron stars," Nature, vol. 259, no. 5542, pp. 377-378, 1976. 

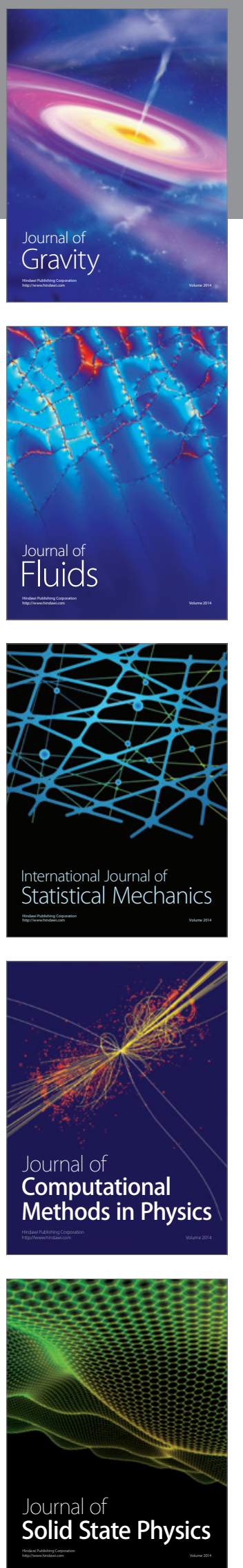

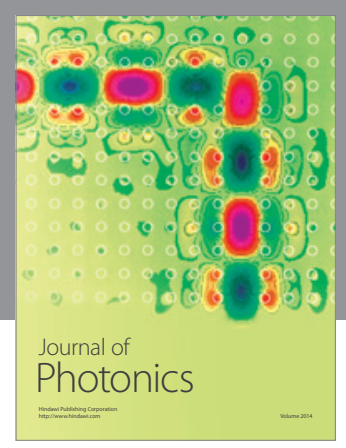

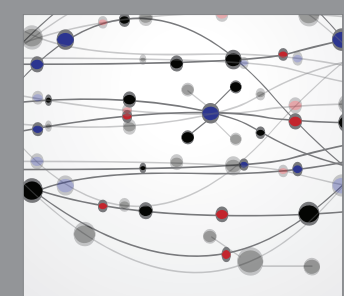

The Scientific World Journal
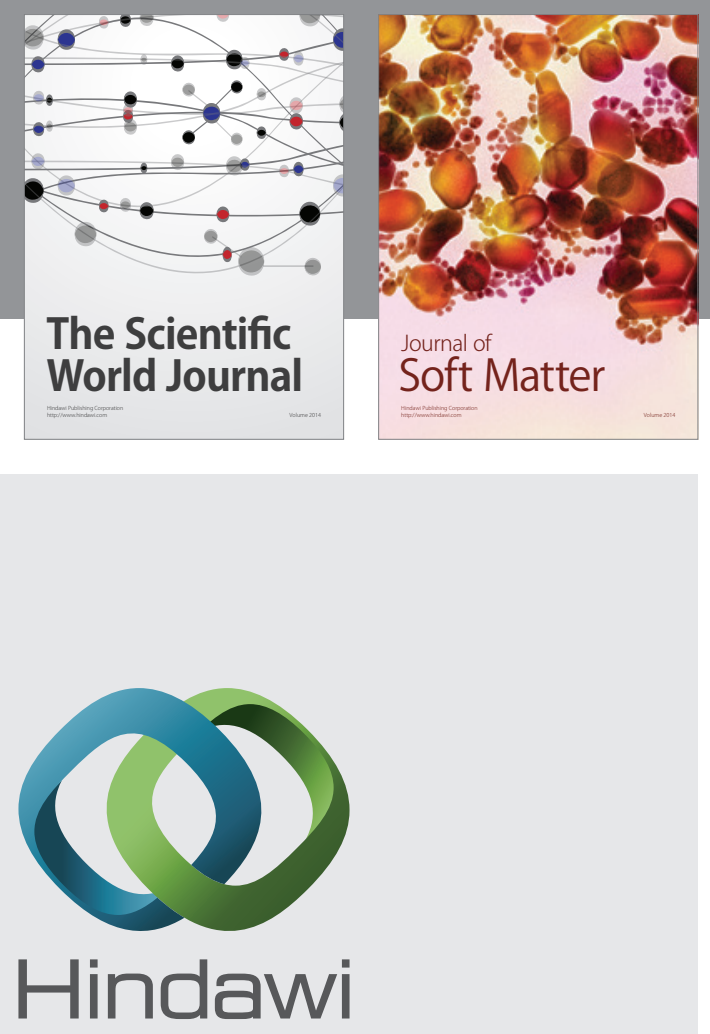

Submit your manuscripts at

http://www.hindawi.com
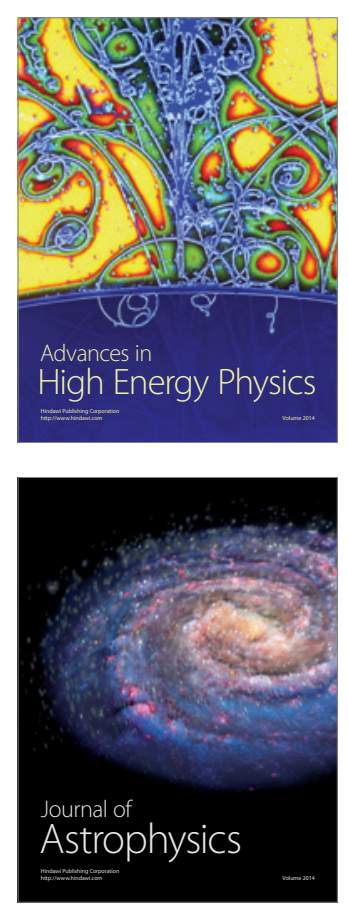
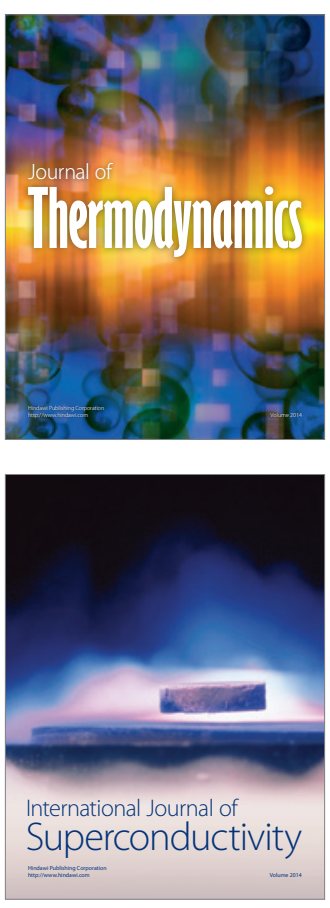
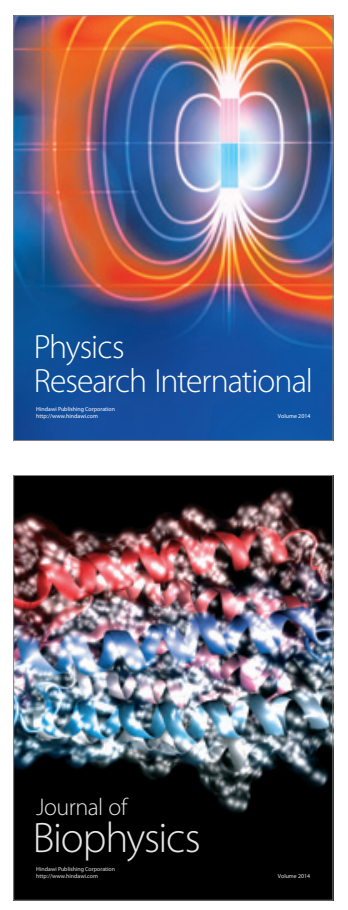
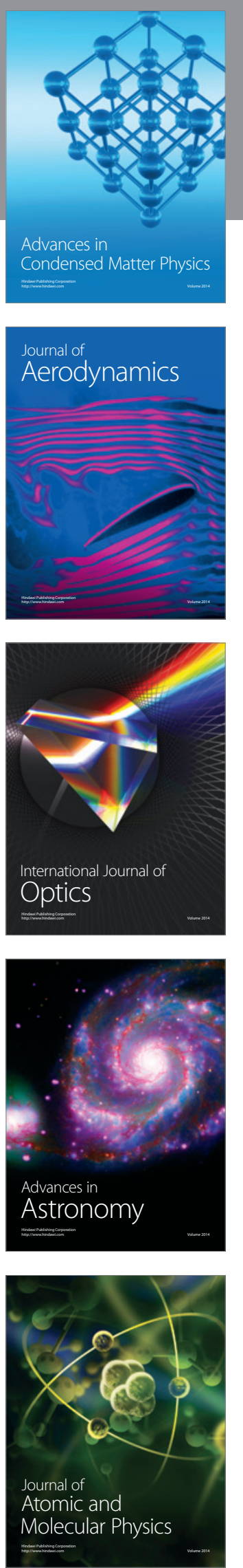